Brit. F. vener. Dis. (1970) 46, 426

\title{
Correspondence
}

\section{Skin biopsy in cases of secondary syphilis}

To the Editor, British fournal of Venereal Diseases

SIR Immunofluorescent techniques have been used to study the skin lesions of pemphigus, pemphigoid, lupus erythematosus, and allergic vasculitis (Kay and Tuffanelli, 1969; Copeman, 1970). In vasculitis of both the necrotizing and lymphocytic types, biopsies of skin lesions have shown immune complexes fixed to the vessel walls (Copeman, 1970). The skin lesions of secondary syphilis are characterized by a vasculitis, with endothelial proliferation and a perivascular infiltrate composed of lymphocytes and plasma cells (Lever, 1967). Secondary lues is also characterized by high levels of antibody against Treponema pallidum and against the cardiolipin used in nonspecific serological tests for syphilis. We considered the possibility that antigen-antibody complexes might be present in the vessel walls in secondary syphilis and play a role in the pathogenesis of the skin lesions of this disease.

Specimens of skin were studied from five seropositive patients with typical papular lesions of secondary syphilis. The tissue was obtained with a 4-mm. skin punch after alcohol preparation and local lidocaine anaesthesia, and then frozen in embedding compound (OCT) with dry ice. The specimens were stored a maximum of 7 days at $-20^{\circ} \mathrm{C}$. until processed. The biopsies were then orientated and cut on a cryostat at $-20^{\circ} \mathrm{C}$. to $6-8$ microns. Sections were rinsed twice in phosphate-buffered saline (PBS) pH 7.4 to remove the OCT embedding medium, and then incubated at room temperature in a moisture chamber for 30 minutes (with various fluorescein-conjugated anti-sera.) The anti-sera employed were fluorescein-isothiocyanate conjugated goat anti-human IgG, IgA, and IgM obtained from Hyland Laboratories. The sera obtained were in lyophilized form and were reconstituted with PBS pH 7.4. After incubation, the slides were rinsed in PBS three times, and then air-dried for 20 minutes before coverslips were applied with glycerin-phosphate buffer $\mathrm{pH} 7 \cdot 4$. The sections were then refrigerated and examined several hours later. A Leitz fluorescent microscope was used equipped with a high pressure Mercury arc lamp (CS $150 \mathrm{~W}$, Type 103803), 2 UV filters (2mm. UG1, $4 \mathrm{~mm}$. UG1), and a Rillenstreuscheibe filter. The specimens were also examined after routine staining with haematoxylin and eosin.

All five specimens had the typical histological appearance of secondary syphilis. However, none showed immunofluorescent staining of vessels or other cutaneous structures. Perhaps, with a larger group of patients or with different immunofluorescent techniques, globulin binding to vessels might be demonstrated in the lesions of secondary syphilis. However, because of the negative findings in all subjects in this small series, we doubt that antigenantibody complexes are involved in the production of the vasculitis of secondary syphilis.

Sincerely,

EDGAR B. SMITH, M.D.

J. KENT BARTRUFF, M.D.

VICTORY BLANCHARD

Department of Dermatology

and Syphilology,

Baylor College of Medicine,

1200 Moursund Avenue,

Houston, Texas 77025,

U.S.A.

April 24, 1970

\section{References}

Copeman, P. W. M. (1970) Brit. F. Derm., 82, Supplement 5, 'The Microvasculature of the Skin', ed. T. J. Ryan, p. 51

Kay, D. M., AND TuffaneliI, D. L. (1969) Ann. intern. Med., 71, 753

LEVER, W. F. (1967) 'Histopathology of the Skin', 4th ed., p. 322. Lippincott, Philadelphia 